\title{
A MAGNETOHYDRODYNAMIC STUDY OF BEHAVIOR IN AN ELECTROLYTE FLUID USING NUMERICAL AND EXPERIMENTAL SOLUTIONS
}

\begin{abstract}
L. P. Aoki,
M. G. Maunsell, and H. E. Schulz

Universidade de São Paulo Escola de Engenharia de São Carlos Departamento de Engenharia Mecânica Parque Arnold Schimidt CP. 13566-590, São Carlos, São Paulo, Brasil lucianoaoki@gmail.com

\section{ABSTRACT}

This article examines a rectangular closed circuit filled with an electrolyte fluid, known as macro pumps, where a permanent magnet generates a magnetic field and electrodes generate the electric field in the flow. The fluid conductor moves inside the circuit under magnetohydrodynamic effect (MHD). The MHD model has been derived from the Navier Stokes equation and coupled with the Maxwell equations for Newtonian incompressible fluid. Electric and magnetic components engaged in the test chamber assist in creating the propulsion of the electrolyte fluid. The electromagnetic forces that arise are due to the cross product between the vector density of induced current and the vector density of magnetic field applied. This is the Lorentz force. Results are present of 3D numerical MHD simulation for newtonian fluid as well as experimental data. The goal is to relate the magnetic field with the electric field and the amounts of movement produced, and calculate de current density and fluid velocity. An u-shaped and $\mathrm{m}$-shaped velocity profile is expected in the flows. The flow analysis is performed with the magnetic field fixed, while the electric field is changed. Observing the interaction between the fields strengths, and density of the electrolyte fluid, an optimal configuration for the flow velocity is determined and compared with others publications.
\end{abstract}

\section{NOMENCLATURE}

A magnetic vector potential

B magnetic flux density, $\mathrm{T}$

E electric field, V/m

F volumetric Lorentz force, $\mathrm{N} / \mathrm{m}^{3}$

$\mathrm{H}$ channel height, $\mathrm{m}$

I current, A

J current density, $\mathrm{A} / \mathrm{m}^{2}$

L channel length, $\mathrm{m}$

Lm magnet length, $m$

$\mathrm{P}$ pressure, $\mathrm{N} / \mathrm{m}^{2}$

$\mathrm{u}$ local velocity, $\mathrm{m} / \mathrm{s}$

u local velocity of the conductor, $\mathrm{m} / \mathrm{s}$

$\mathrm{V}$ power supply voltage, $\mathrm{V}$

W channel width, $\mathrm{m}$

\section{Greek symbols}

$\begin{array}{ll}\varepsilon_{0} & \text { vacuum permittivity } \\ \eta & \text { kinematic viscosity, } \mathrm{m}^{2} / \mathrm{s} \\ \mu & \text { dynamic viscosity, } \mathrm{kg} / \mathrm{m} . \mathrm{s} \\ \mu_{0} & \text { vacuum permeability } \\ \phi & \text { electrical vector potential, } \mathrm{V} / \mathrm{m} \\ \rho & \text { density, } \mathrm{kg} / \mathrm{m} \\ \rho_{e} & \text { total charge density, } \mathrm{C} / \mathrm{m}^{2} \\ \sigma & \text { electrical conductivity, }{\mathrm{S} . \mathrm{m}^{-1}}^{2}\end{array}$

Keywords: macro pumps, magnetohydrodynamic, Lorentz force

\section{INTRODUCTION}

From principles of electricity and magnetism we know that magnetic fields interact directly with many artificial and natural fluids. They are used in industries to heat, cool, pump and levitate liquid metals and to generate Earth's magnetic field, which is maintained due the movement of the fluid in its core. This phenomenon where a fluid interacts with magnetic field is called magnetohydrodynamics or simply MHD.

Ritchie (1832) was the first to discover the MHD phenomenon. He described the basic operation principles of a MHD pump, where an electric current and a magnetic field pass through an electrolyte solution. MHD is concerned with the mutual interaction of fluid flow and magnetic fields. The fluids must be electrically conducting and nonmagnetic, which limits us to liquids metals, hot ionized gases (plasmas) and electrolytes.

According to Davidson (2001) the mutual interaction of a magnetic field $\mathrm{B}$, and velocity field $\mathrm{u}$, arises partially as a result of the laws of Faraday and Ampère, and partially because of the Lorentz force experienced by a current-carrying body.

Shercliff (1965), says that and conductor, in the presence of a varying magnetic field, for example, a moving magnets or a solenoid powered by a power supply varying current, create a induced electric 
current density in this conductor which interacts with the original magnetic field, resulting in electromagnetic forces that change de fluid's pressure gradient or the state of movement of fluid.

The experiment discussed here consisted in a rectangular circuit (known as macropump) whose main purpose is to pump an electrolyte solution. From the flow movement it is possible to obtain a velocity profile over the channel and associate it with the MHD phenomenon.

\section{The MHD Phenomenon Investigations}

Many studies about velocity profiles were made, including a recent work of Andreev et al. (2004) which shows an experimental study of liquid metal conductor on the influence of non homogeneous magnetic fields. Unlike a macropump, this acts like a flow brake and it is applicable in mining industries.

Ramos and Winovich (1990) applied finite element method to simulate a MHD channel flows as a function of the Reynolds number and wall conductivity. Lemoff and Lee (2000) applied a computational method to describe a micro fluidic pump using alternated current MHD propulsion to propel an electrolyte solution. Following this research line the author held the experimental and computational MHD phenomenon using salty water.

\section{THEORETICAL ANALYSIS}

The formulation of the MHD steady state model has been derived from the Maxwell's equations (electromagnetic domain) coupled with NavierStokes equations. Therefore the model is governed by the electromagnetism and fluid dynamics equations:

$$
\begin{gathered}
\nabla \cdot \mathbf{E}=\frac{\rho_{e}}{\varepsilon_{0}} \quad(\text { Gauss law) } \\
\nabla \times \mathbf{E}=-\frac{\partial \mathbf{B}}{\partial t} \quad \text { (Faraday law) } \\
\nabla \cdot \mathbf{B}=0 \quad \text { (No magnetic monopoles) } \\
\nabla \times \mathbf{B}=\mu_{0} \mathbf{J}+\mu_{0} \varepsilon_{0} \frac{\partial \mathbf{E}}{\partial t} \quad \text { (Ampère law) }
\end{gathered}
$$

where Eq. (1), Eq. (2), Eq. (3) and Eq. (4) represents the Maxwell's equations. Using the divergence and applying the Gauss Law in Eq. (4) we can obtain:

$$
\nabla \cdot \mathbf{J}=-\varepsilon_{0} \frac{\partial}{\partial t} \nabla \cdot \mathbf{E}=-\frac{\partial \rho_{e}}{\partial t} \text { or } \nabla \cdot \mathbf{J}=0
$$

In MHD problems, the second term $\frac{\partial \rho_{e}}{\partial t}$ is negligible for a conductor. Using the Ohm's Law relations and the Ampère's Law again:

$$
\begin{gathered}
\nabla \times\left(\frac{\nabla \times \mathbf{A}}{\mu_{0}}\right)=\mathbf{J} \text { or } \nabla \times \mathbf{B}=\mu_{0} \mathbf{J} \\
\mathbf{J}=\sigma\left[-\nabla \phi+\mathbf{u}_{1} \times(\nabla \times \mathbf{A})\right]
\end{gathered}
$$

Where the electromagnetic domain or (EMD), is represent by the Maxwell-Ampère law on the Eq. (6), Ohm's law, Eq. (7) and the conservation of the electrical current Eq. (5), where $\mathbf{J}$ is the total current density, $\mathbf{A}$ is the magnetic vector potential, $\mu$ is the permeability, $\sigma$ is the electrical conductivity of salty water, $\mathbf{u}_{1}$ is the velocity of an electrical particle and $\phi$ is the electrical vector potential. For an incompressible Newtonian fluid we have:

$$
\begin{gathered}
\frac{\partial \mathbf{u}}{\partial t}+\left(\mathbf{u}_{2} \cdot \nabla\right) \mathbf{u}_{2}= \\
\frac{1}{\rho} \nabla P+\eta \nabla^{2} \mathbf{u}_{2}+\frac{1}{\rho}(\mathbf{J} \times \mathbf{B})(8) \\
\nabla \cdot \mathbf{u}_{2}=0
\end{gathered}
$$

Where $\mathbf{u}_{2}$ is the local fluid's velocity, $\nabla P$ is the gradient pressure, $\eta$ is the kinematic viscosity of the salty water and $\rho$ is the density. However in a stationary conductor it is found that the current density $\mathbf{J}$, is proportional to the force experienced by the free charges. This is reflected in Ohm's Law, $\mathbf{J}=\sigma \mathbf{E}$. In a conducting fluid the same law applies, but now we must use the electric field measured in a frame moving with the local velocity of the conductor, so $\mathbf{u}_{1}=\mathbf{u}_{2}=\mathbf{u}$. The electrical scalar potential $\phi$ can be determined by solving the Poisson equation:

$$
\nabla^{2} \phi=\nabla \cdot[\mathbf{u} \times(\nabla \times \mathbf{A})]
$$

Here the space depended variables are $\mathbf{A}(x, y, z), \mathbf{J}(x, y, z)$ e $\phi=(x, y, z)$ and Eq. (7) can be also formulated in terms of $\mathbf{E}=-\nabla \phi$ and $\mathbf{B}=(\nabla \times \mathbf{A})$ bringing to:

$$
\mathbf{J}=\sigma(\mathbf{E}+\mathbf{u} \times \mathbf{B})
$$

The coupling between the electromagnetic model and the fluid model is achieved by introducing the Lorentz force $\mathbf{F}$, given by $\mathbf{J} \times \mathbf{B}$ where $\mathbf{B}$ is the external magnetic field imposed by the magnets. 


\section{THE MHD PROBLEM}

The MHD analysis occurred in two stages, experimental and computational. The configurations and initial conditions observed in laboratory were inserted in the computational model.

\section{Experimental Phase}

The goal is to simulate an electromagnetic pump using the principles of MHD and from that obtain the pressure and velocity data and analyze the flow profile. For that purpose an oval circuit was built Fig. 1 , and filled with electrolyte solution (salty water). The (EMD) was created according to Fig. 2.

The EMD is composed by two strong neodymium magnets $N d F e B$ (grade N35 and $\mathrm{B}_{0}=0.3 \mathrm{~T}$ ) with dimensions of $70 \mathrm{~mm} \times 20 \mathrm{~mm} \times$ $10 \mathrm{~mm}$ and two electrodes with $20 \mathrm{~mm}$ x $20 \mathrm{~mm} \times 3$ $\mathrm{mm}$. The experiment proceeded by connecting the electrodes to a Power supply and switching the voltage between 12 Volts and 30 Volts.

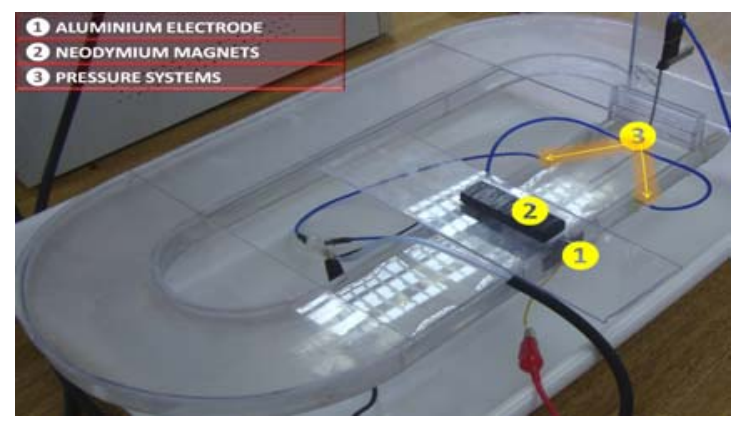

Figure 1. MHD circuit.

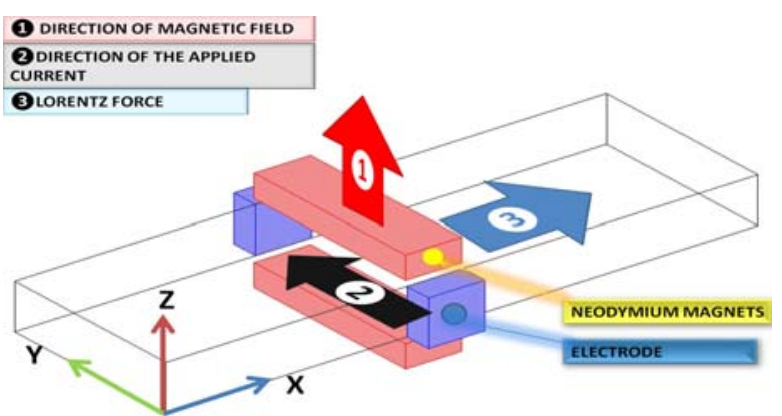

Figure 2. Electromagnetic domain (EMD).

As mentioned earlier the interaction of electric and magnetic fields results in the Lorentz's electromagnetic force that moves the fluid.

Using a differential manometer, which captures the differences between the static and total pressure, you can obtain the local velocities of fluid in different positions of the channel and plot comparison graphs, according to Figs. 4-6. For better results we analyzed the velocity profiles along the channel width (y axis) for different points along the $\mathrm{x}$ axis. The Pitot, which dimensions was $1.5 \mathrm{~mm}$ of diameter (to avoid salt particles and electrolyte wastes), was set at t $9 \mathrm{~mm}$ depth in the water layer of $18 \mathrm{~mm}$.

It is important to say that the Pitot was not installed exactly on the channel's edges, but immediately close, due the positioning difficulties and the geometry. The experiment took place only in the EMD, i.e., near the electrodes and magnets region. In Fig. 3 the EMD is found in the middle of channel (approximately $0.14 \mathrm{~m}$ ). The reason to exclude the rest of the circuit is that it will not have the MHD influence whatsoever. The graphics and pictures are shown below:

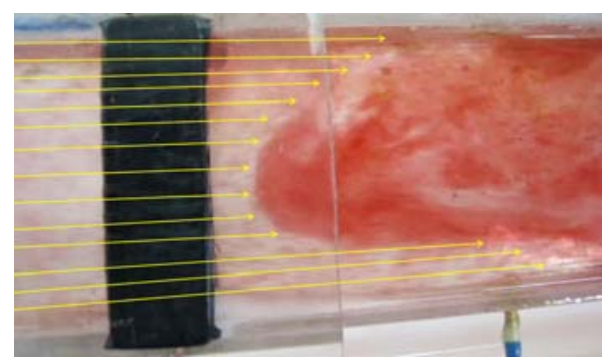

Figure 3. Fluid u-shaped velocity profile along the $y$ axis at 30 Volts. A dye was used for better visualization.

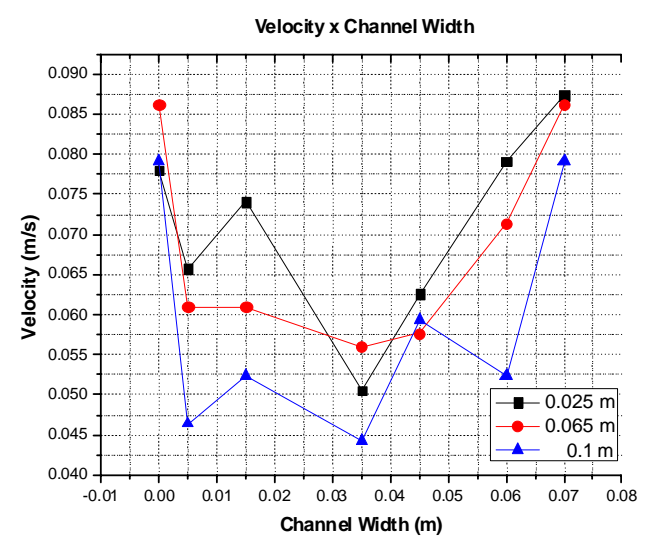

Figure 4. Mean fluid velocity profile along the $y$ axis for different $\mathrm{x}$ positions at 12 Volts.

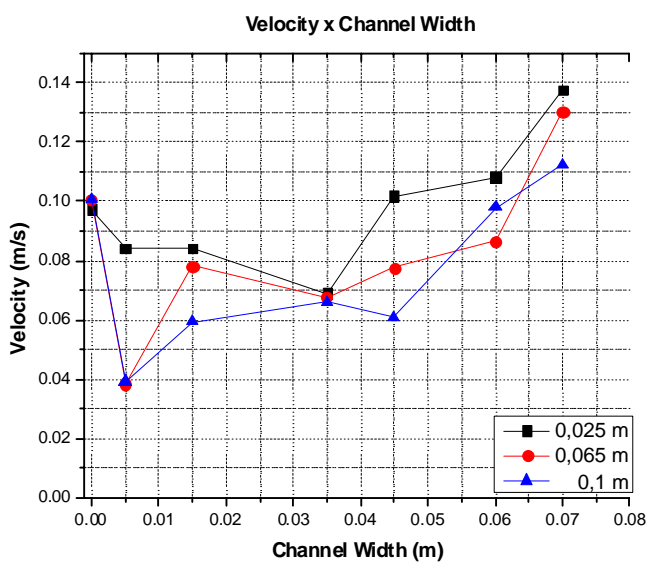

Figure 5. Mean fluid velocity profile along the $y$ axis for different $\mathrm{x}$ positions at 20 Volts. 


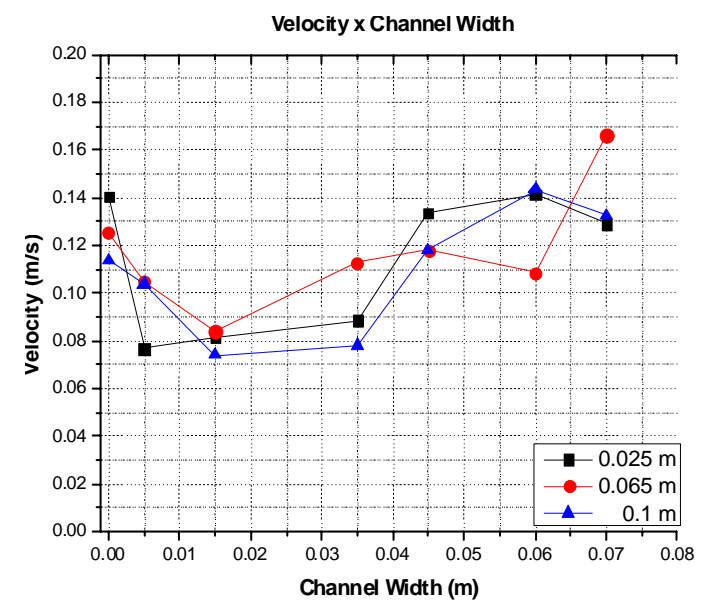

Figure 6. Mean fluid velocity profile along the $y$ axis for different $\mathrm{x}$ positions at 30 Volts.

\section{Computational Phase}

The computational model was obtained according to the laboratory's experimental data and showed in Fig. 7. An electrically isolated rectangular channel is filled with an electrolyte solution.

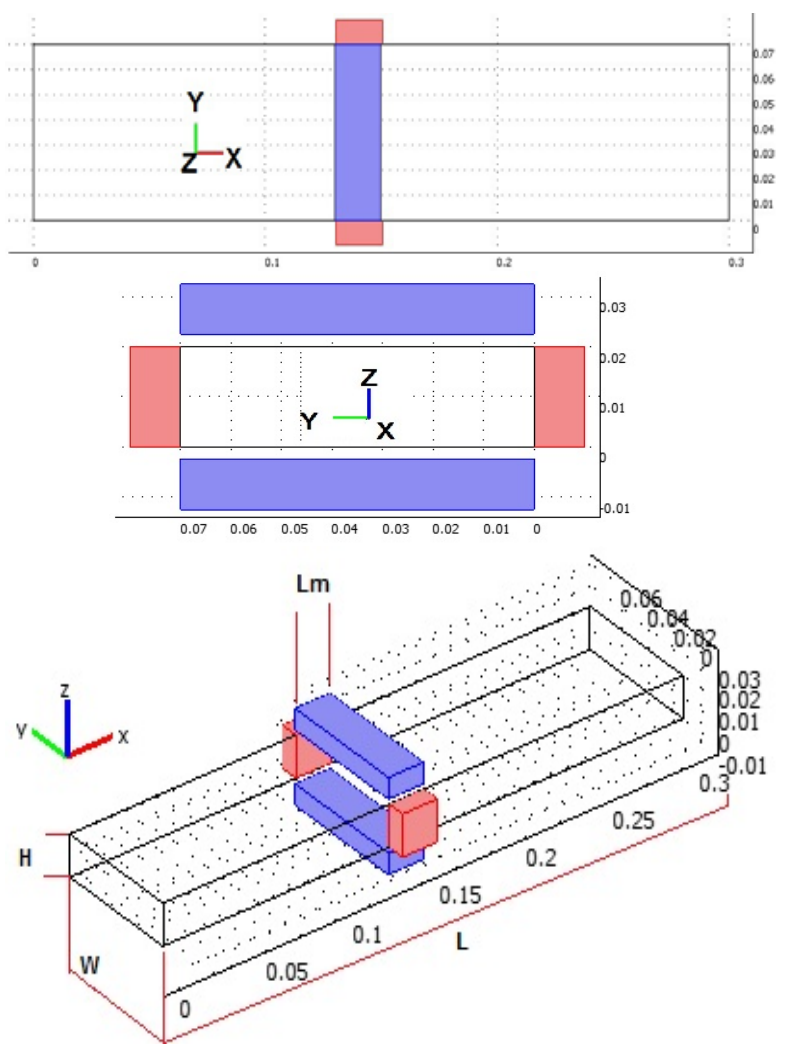

Figure 7. MHD Domain the channel has length $\mathrm{L}=$ $0.3 \mathrm{~m}$, height $\mathrm{H}=0.02 \mathrm{~m}$ and width $\mathrm{W}=0.07 \mathrm{~m}$. The magnet has length $\mathrm{Lm}=0.02 \mathrm{~m}$.

The channel is subjected to an externally magnetic field, perpendicular to the fluid, produced by neodymium magnets. The channel's plane and therefore the whole circuit is located at horizontal plane $x-y$ and $z=0$. The fluid is set to have the same conditions of salty water like density and dynamic viscosity. An external electric field is applied along the circuit, simulating the real power supply. The EMD is delimitated by an air sphere, representing the real experimental conditions. Some initial and boundary conditions were imposed to simulate de MHD problem. The 3D MHD equations was solved using the finite element method in COMSOL multiphysics $3.5 \AA$. The conditions are presented in Table 1.

Table 1. Process parameters.

\begin{tabular}{|c|c|}
\hline Equation Type & Boundary Conditions \\
\hline Electromagnetics & $\begin{array}{l}\mathrm{B}(\mathrm{x} ; \mathrm{y} ; 0.03)=\mathrm{B}_{\mathrm{z}}(+) 0.13<\mathrm{x}< \\
0.15 ; 0<\mathrm{y}<0.07 \\
\mathrm{~B}(\mathrm{x} ; \mathrm{y} ;-0.13)=\mathrm{B}_{\mathrm{z}}(-) 0.13<\mathrm{x}< \\
0.15 ; 0<\mathrm{y}<0.07 \\
\text { Electrodes } \\
\phi(\mathrm{x} ; 0.08 ; \mathrm{z})=\mathrm{V}(+) 0.13<\mathrm{x}< \\
0.15 ; 0<\mathrm{z}<0.02 \\
\phi(\mathrm{x} ;-0.01 ; \mathrm{z})=\mathrm{V}(-) 0.13<\mathrm{x}< \\
0.15 ; 0<\mathrm{z}<0.02 \\
\text { Insulations } \\
\text { Magnetically insulated elsewhere } \\
\text { Electrically insulated elsewhere }\end{array}$ \\
\hline Fluid Dynamics & $\begin{array}{l}\mathrm{U}(\mathrm{x} ; 0 ; \mathrm{z})=0 \text { (no-slip), } \mathrm{U}(\mathrm{x} ; \mathrm{y} ; \\
0)=0 \text { (no-slip) } \\
\mathrm{U}(\mathrm{x} ; 0.07 ; \mathrm{z})=0 \text { (no-slip), } \mathrm{U}(\mathrm{x} ; \\
\mathrm{y} ; 0.02)=0 \text { (no-slip) } \\
\text { Inlet } \\
\mathrm{U}(0 ; \mathrm{y} ; \mathrm{z})=2 \mathrm{U}_{0} * \mathrm{U}^{*} 1 * \mathrm{~s} 1 *(1- \\
\mathrm{s} 1)^{*} \mathrm{~s} 2 *(1-\mathrm{s} 2) ; 0<\mathrm{y}<0.07 ; 0<\mathrm{z} \\
<0.02 \\
\text { Outlet } \\
\mathrm{P}(0.3 ; \mathrm{y} ; \mathrm{z})=0 ; 0<\mathrm{y}<0.07 ; 0< \\
\mathrm{z}<0.02 \\
\text { Body force }=\mathrm{F}_{1}=\text { Lorentz force }\end{array}$ \\
\hline
\end{tabular}

The parameters $\mathrm{U}_{0}$ and $\mathrm{U}$ represents de maximum and mean velocities respectively, and the arc length parameters $\mathrm{s} 1$ and $\mathrm{s} 2$ creates a 3D parabolic velocity profile on an inflow boundary. The inlet velocity conditions in Fig. 8 was based in a function that describes the real velocity in experiment, since the velocity is faster in the edge than in inner channel due the geometry.

Due to the coupling between electromagnetic and fluid dynamics equations, an iterative solution is used. First, the magnetic components have been given by the externally imposed magnets are solved to determine the magnetic flux density $\mathbf{B}$. Next the electrical potential $\phi$, the electric field $\mathbf{E}$, and the current density $\mathbf{J}$ are determined by solving the Poisson equation and Ohm's law, Eq. (9) and Eq. (10). In the first run, the component of $\mathbf{J}, \mathbf{u} \times \mathbf{B}$ is zero, because the fluid is stopped. After the product of $\mathbf{J} \times \mathbf{B}$ is evaluated and added as Lorentz force in 
the Navier - Stokes equation Eq. (8). From Eq. (8), the velocity is finally determinated and reevaluated on Eq. (10) thus completing the iteration cycle.

For simplicity in most of MHD problems we shall assume that the time-dependent flow $\partial \mathbf{u} / \partial t=0$ has travelled sufficiently far down de duct ( $x$ direction) to have reached an $\mathrm{x}$-independent form, so $\mathbf{u} \cdot \nabla \mathbf{u}=0$ and $\mathbf{u}=\mathbf{u}(y, z)$, and we assume that gravitational forces are unimportant. Then the flow's steady state equation of motion takes the form $\nabla P=\mathbf{J} \times \mathbf{B}+\eta \nabla^{2} \mathbf{u}$. Here we can calculate de pressure gradient. Another way to find the velocity is using the equation based on the difference between the total and static pressures considering the density and dynamic viscosity of the fluid.

Taking advantages of computer simulations, the author created Lorentz force's profiles acting in many parts of the channel in function of voltages, magnetic flux density (Figs. 9 and 10) and the current density along the circuit (Figs. 20-22). It was expected from Figs. 11, 13 and 15 a slight decrease of the Lorentz electromagnetic forces proving the acceleration near the wall.

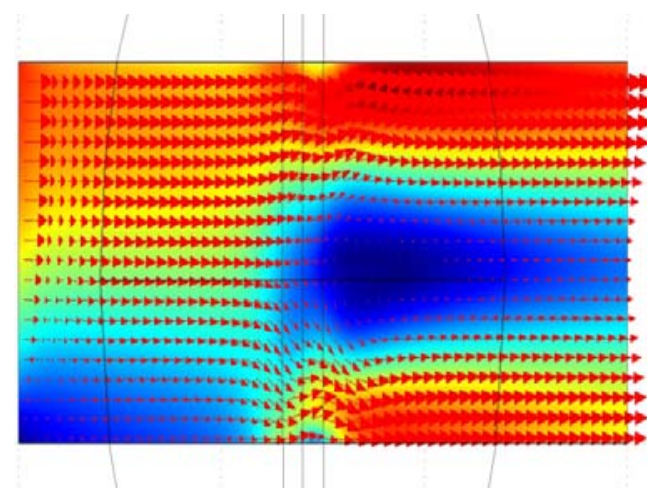

Figure 8 . Vectors of velocity field in the axial horizontal plane $(\mathrm{z}=0)$ of the channel.

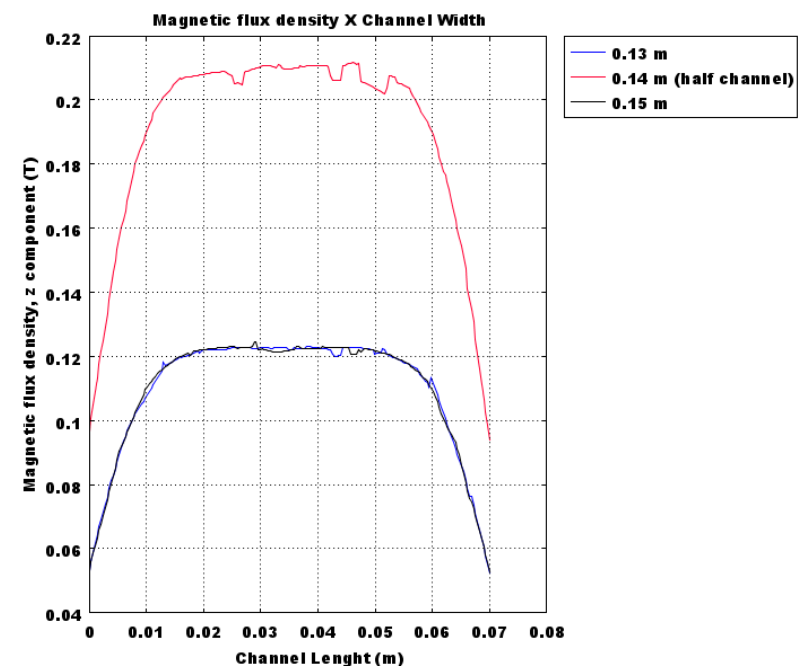

Figure 9. Magnetic flux density along the y axis for different $\mathrm{x}$ positions.
In order to corroborate the experimental method, computational simulations were carried based on real initial and boundary conditions. Similar to the experimental data, the computational data (velocity profiles along the channel) were plotted in Figs. 12, 14 and 16, proving a slight change in the velocities curves on the channel's wall, and revealing and M-shape profile. Figures 17-19 shows a comparison between experimental and computational data.

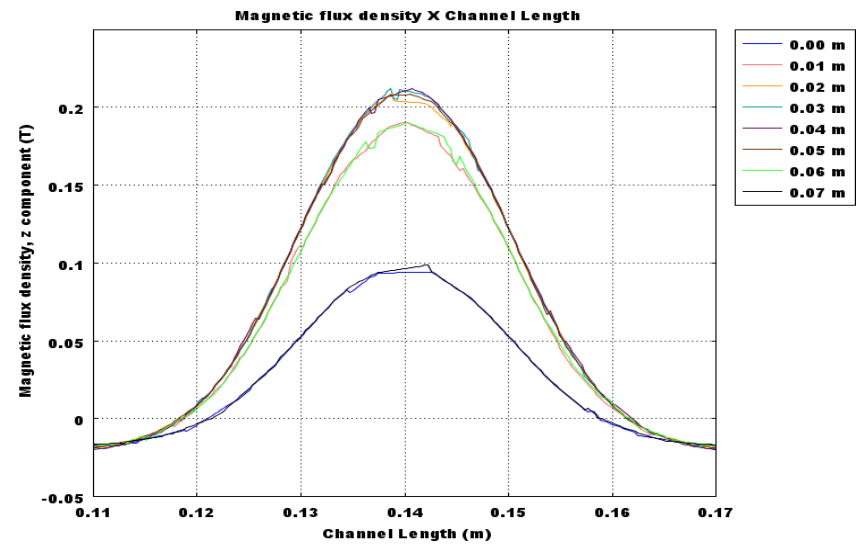

Figure 10. Magnetic flux density along the $\mathrm{x}$ axis for different y positions.

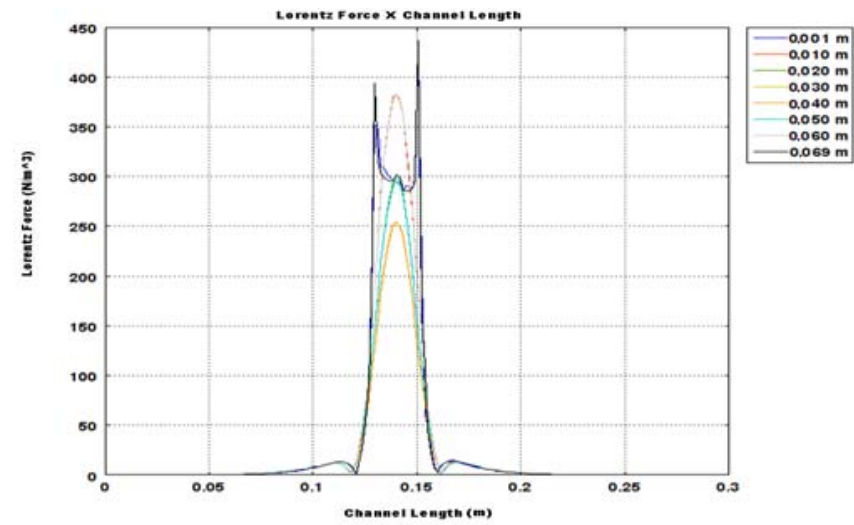

Figure 11. Lorentz force along the $\mathrm{x}$ axis for different $\mathrm{y}$ positions at 12 Volts.

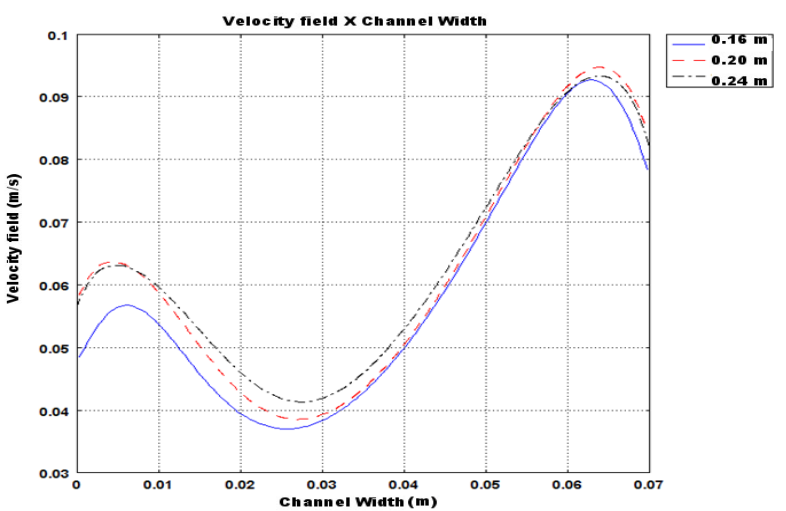

Figure 12. Fluid velocity profile along the y axis for different $\mathrm{x}$ positions at 12 Volts. 


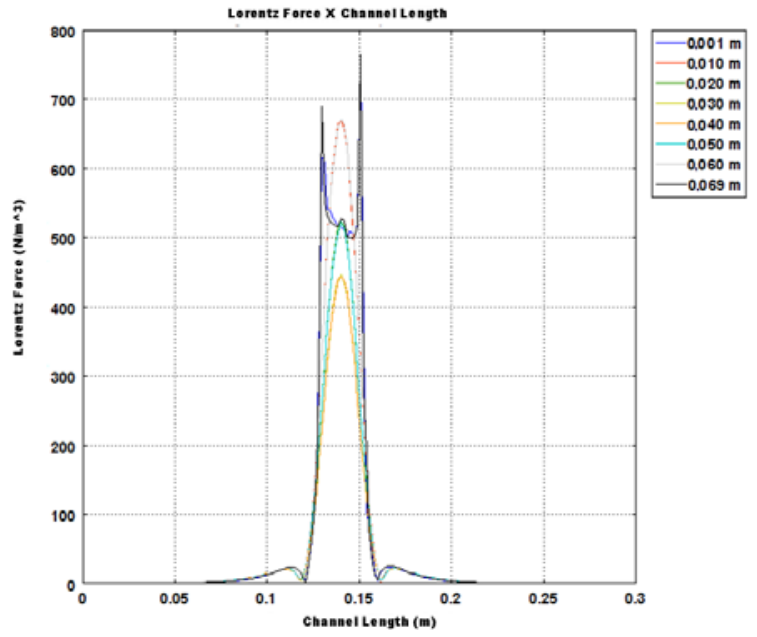

Figure 13. Lorentz force along the $\mathrm{x}$ axis for different $\mathrm{y}$ positions at 20 Volts.

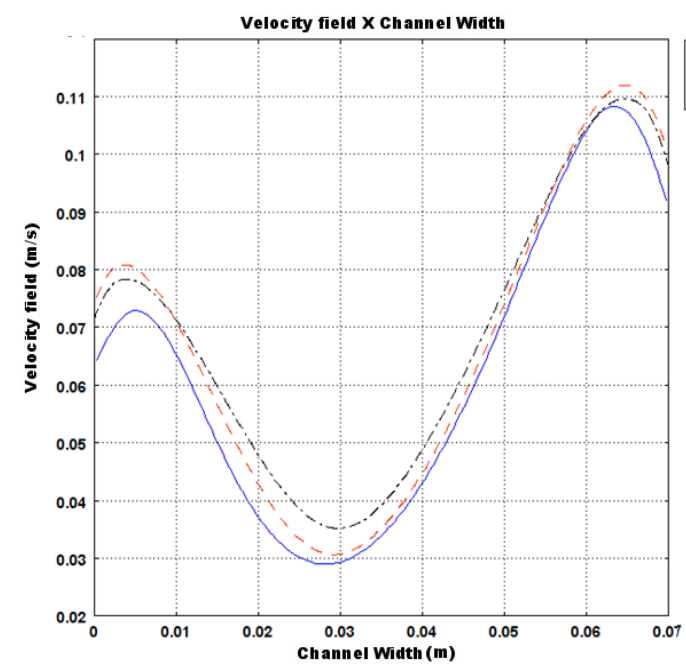

Figure 14. Fluid velocity profile along the y axis for different $\mathrm{x}$ positions at 20 Volts.

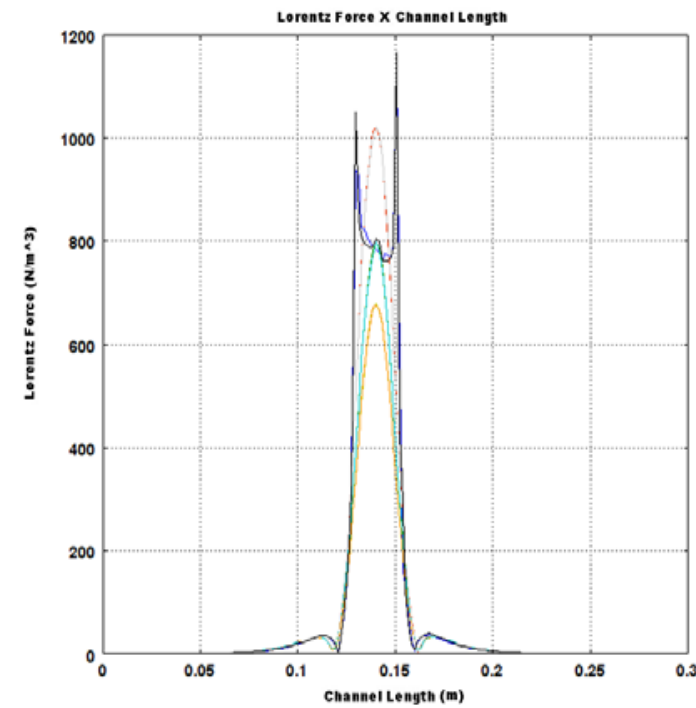

Figure 15. Lorentz force along the $\mathrm{x}$ axis for different y positions at 30 Volts.

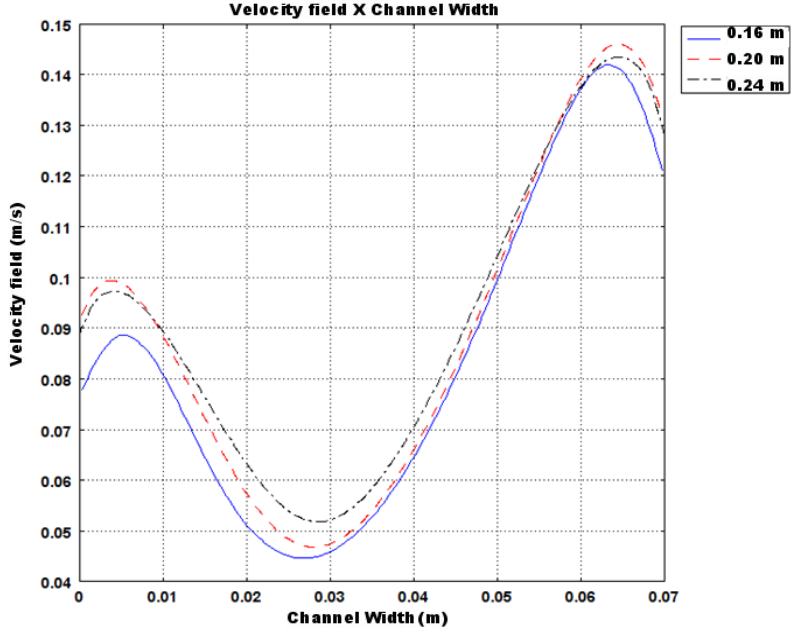

Figure 16. Fluid velocity profile along the y axis for different $\mathrm{x}$ positions at 30 Volts.

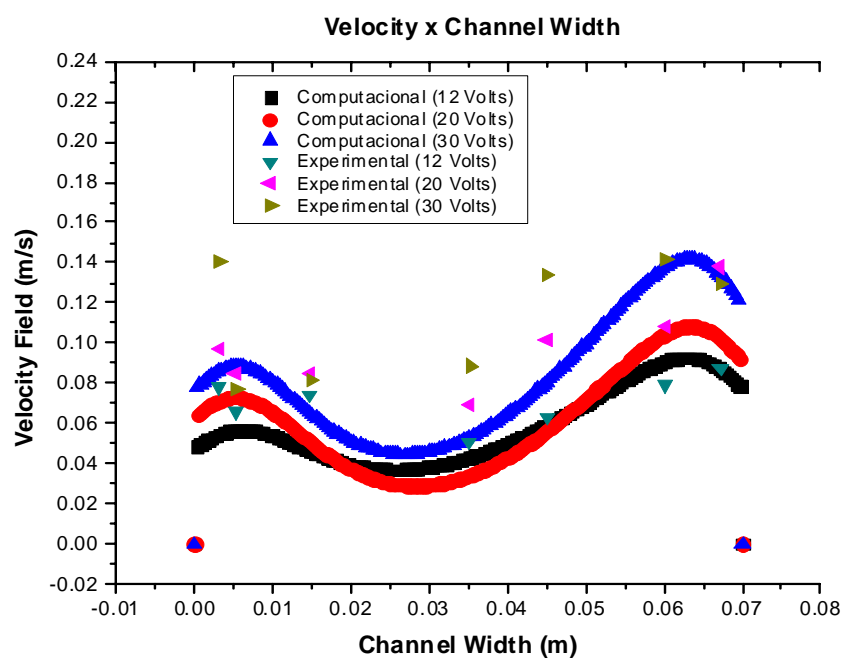

Figure 17. Experimental and computational velocity profiles for different $\mathrm{y}$ positions at $\mathrm{x}=0.16 \mathrm{~m}$.

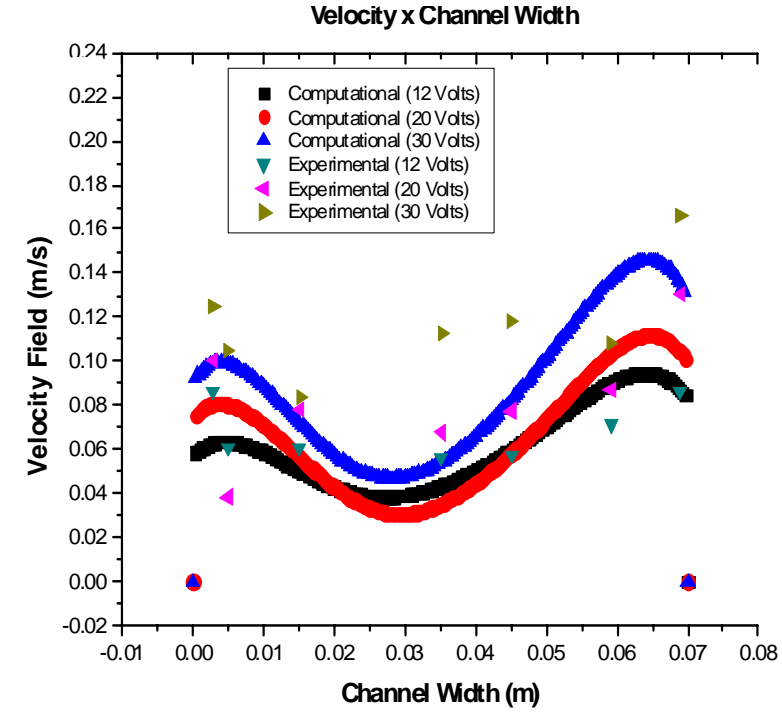

Figure 18. Experimental and computational velocity profiles for different $\mathrm{y}$ positions at $\mathrm{x}=0.20 \mathrm{~m}$. 


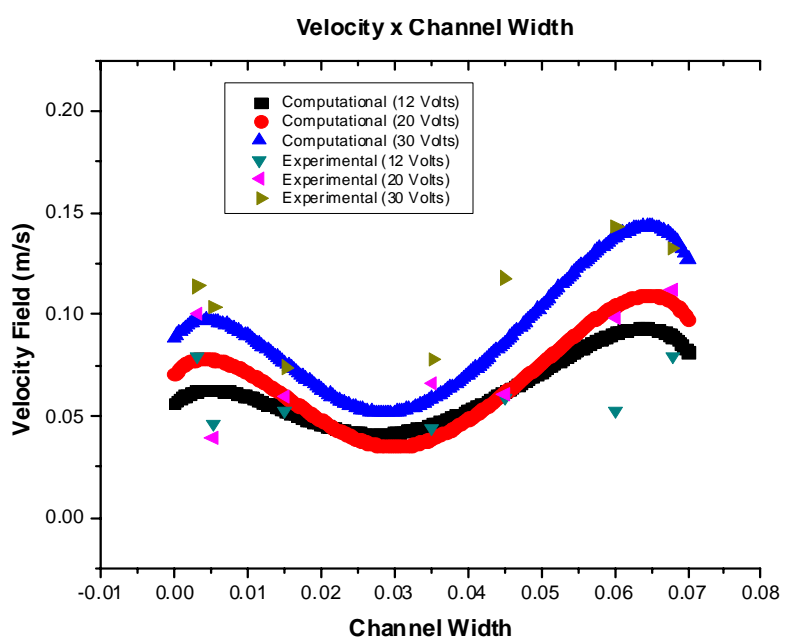

Figure 19. Experimental and computational velocity profiles for different $\mathrm{y}$ positions at $\mathrm{x}=0.24 \mathrm{~m}$.

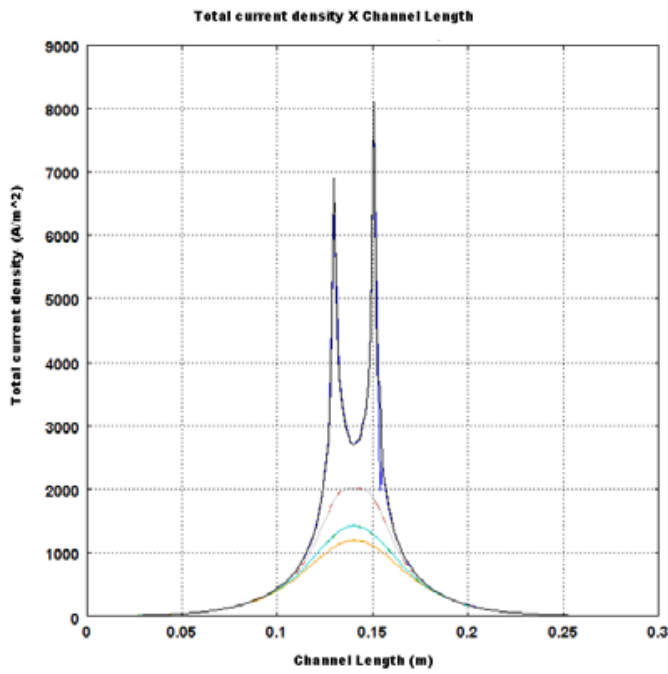

Figure 20. Total current density along the $\mathrm{x}$ axis for different y positions at 12 Volts.

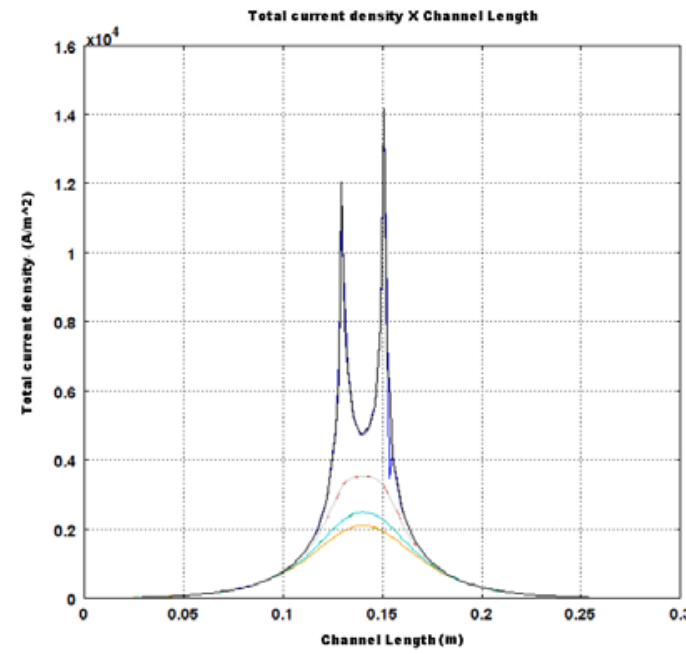

Figure 21. Total current density along the $\mathrm{x}$ axis for different y positions at 20 Volts.
Figure 23 shows the electromagnetic forces acting on the fluid. Note that the Lorentz forces are more strong and concentrated in the borders.

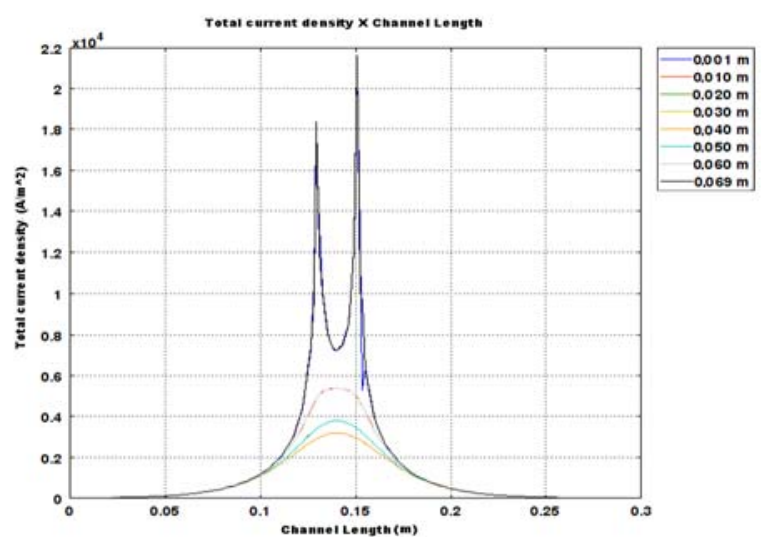

Figure 22. Total current density along the $\mathrm{x}$ axis for different y positions at 30 Volts.

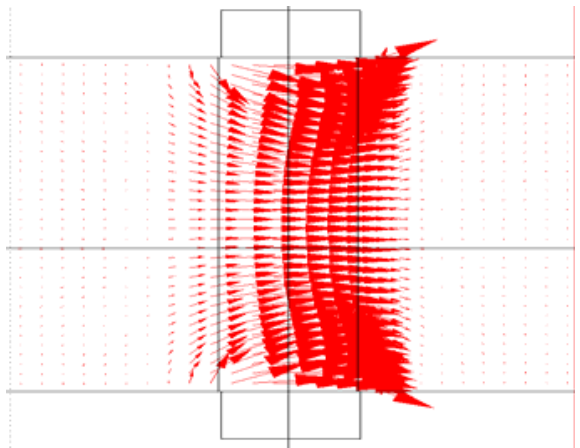

Figure 23. Electromagnetic forces acting on the fluid.

\section{CONCLUSIONS}

In this work, experimental and computational analysis were made to explain the MHD phenomenon. In the experimental part, local velocity measurements were recorded for three different voltages, and profile graphics along the channel were plotted, revealing an unusual behavior; velocities profiles tend to accelerate near the channel's walls, while in the middle, the opposite happens. In some cases the velocity increases significantly when switch the voltage, thanks to Lorentz electromagnetic force (Fig. 2), which is more powerful in the wall region and pumps the fluid with more power. To maintain the momentum conservation, the velocity in the middle of the channel has to be slower than the wall region. A good review on the MHD equations reveals some tricks. It's important to note that the velocity not only depends on voltage, but the fluid's conductivity and magnetic fields, which causes a significantly change in the velocity profile.

The profiles can be compared to Daod and Kandev (2008) publication, which a detailed analysis 
was made for a highly conductive molten metal subjected to an inhomogeneous magnetic field. Another curiosity observed is that in one side of the wall, the velocity is larger than the other one. This can be explained by the oval channel's geometry, where the fluid must travel a greater distance in the external wall in comparison to the internal walls to maintain de momentum conservation.

Comparing the experimental and computational velocity profiles we notice a small difference between the results (however in the error's margin) of magnitude of the order of $10^{-2} \mathrm{~m} / \mathrm{s}$ which lies within our expectations.

In the experimental measurement were noticed small oscillations in velocity profile (precisely in the middle of the channel) that can be easily explained. One of the reasons is the MHD phenomenon itself that is very difficult to be measured with all the apparatus present. Another problem found in all experiments, not only in MHD experiments is the errors. We can classify in two types of error basically; a random errors, for example, a human error involving miscalculations, data analysis and the incorrect reading of an instrument; and a systematic errors, for example, improper measuring techniques, defects in instrument (the manometer has a error of reading of $0.1 \mathrm{~Pa}$ ) or not calibrated; changes in temperature, density, fluid's impurities and electrolysis which causes a variation in velocity profiles are some difficulties that we can encounter in experimental analysis and that can change the measurements.

However such measures explained here are valid and satisfactory and present unusual velocity profile patterns which can be applied not only in this paper but in several MHD devices. Recent works presents such velocity profiles: Patel (2009) showed a computational micro pump using a MHD device with the same velocity patterns. Andreev et al (2006) explain the functions of a brake flow, using a similar macropump and showing the same velocity profiles.

Despite appearing similar to a conventional water pump, the MHD pump has no moving mechanical parts, while exhibiting a precise flow control, reduced energy consumption and less dross formation. We can adjust the flow rate and velocity by adjusting the electric and magnetic field values only.

These features can be easily applied in maritime propulsion, bioengineering, mining industry and nuclear engineering, proving that MHD has a promising feature in the future.

\section{ACKNOWLEDGEMENTS}

Aoki.P. L thanks the School of Engineering of São Carlos - University of São Paulo (Department of Electric Engineering), for allowing the use of the COMSOL multiphysics $3.5 \AA$.

\section{REFERENCES}

Andreev, O., Kolesnikov, Y., and Thess, A., 2006, Experimental Study of Liquid Metal Channel Flow Under the Influence of a Nonuniform Magnetic Field, Physics of Fluids, Vol. 18, No. 6, pp. 0651081 - 06510811.

Daou, A., and Kandev, N., 2008, MagnetoHydrodynamic Numerical Study of DC Electromagnetic Pump for Liquid Metal, in: Proceedings of the COMSOL Conference, Hannover, Germany.

Davidson, P., A, 2001, An introduction to Magnetohydrodynamics, Cambridge University Press.

Lemoff, A. V., and Lee, A. P., 2000, An AC Magnetohydrodynamic Micropump, Sensors and Actuators B: Chemical, Vol. 63, No. 3, pp. 178 185(8).

Patel, V. D., 2007, Electroosmosis and Thermal

Effects in Magnetohydrodynamic (MHD) Micropumps Using 3D MHD Equations, A Thesis Presented to the Faculty of San Diego University.

Ramos, I. J., and Winowich, N. S., 1990, Finite

Diference and Finite Elements Methods for MHD

Channel Flows, International Journal for Numerical Methods in Fluids, Vol. 11, No. 6, pp. 907 - 934.

Ritchie, W., 1832, Experimental Researches in Voltaic Electricity and Electromagnetism, Philosophical Transactions of the Royal Society of London, Vol. 122, pp. 279 - 298.

Shercliff, J. A, 1965, Magnetohydrodynamics, Warwick University, England.

Received: September 27, 2012

Revised: October 27, 2012

Accepted: November 27, 2012 